\title{
Human Immunodeficiency Virus-1 and Preconception Care
}

\author{
Margaret A. Lampe
}

Published online: 11 July 2006

(C) Springer Science+Business Media, Inc. 2006

Keywords HIV · Pregnancy · Preconception care · Perinatal

Human immunodeficiency virus (HIV) is a condition that increasingly affects women, especially women of childbearing age. Early in the HIV epidemic, HIV infection and AIDS were diagnosed in relatively few women in the United States. Today, the HIV/AIDS epidemic represents a growing and persistent health threat to women, especially young women and women of color. In 2001 and 2002, HIV infection was the leading cause of death for African American women aged 25-34 years and was among the four leading causes of death for African American women aged 20-24 and 35-44 years, as well as Hispanic women aged 35-44 years [1, 2].

Although HIV is increasing among women, the incidence in children from perinatal transmission is decreasing. With recommendations for routine screening for HIV during pregnancy and the availability of effective interventions to dramatically reduce the risk of perinatal transmission, the number of annual perinatally acquired AIDS cases from 19851994 has declined approximately 95\% [3], representing one of the most striking success stories in the 25-year effort to reduce the impact of HIV/AIDS in the United States.

HIV infection can be transmitted from mother to child in utero, during labor and delivery and through breastfeeding.

Invited brief report for a special supplement on preconception care to the Maternal and Child Health Journal.

M. A. Lampe $(\bowtie)$

U. S. Centers for Disease Control and Prevention,

Division of HIV/AIDS Prevention, Epidemiology Branch,

Mother-Child Transmission Team,

Atlanta, GA, USA

e-mail: MLampe@cdc.gov
In the absence of intervention and without breastfeeding, the risk of perinatal HIV transmission is approximately $25 \%$ (range 13-39\%) [4]. Evidence suggests that in the absence of breastfeeding, 30\% of transmission occurs before birth and $70 \%$ around the time of delivery [4].

In February 1994, the results of the Pediatric AIDS Clinical Trials Group (PACTG) Protocol 076 documented that zidovudine (ZDV) chemoprophylaxis, initiated at 1434 weeks gestation and continued through pregnancy, followed by intravenous ZDV during labor and oral ZDV to the infant for the first 6 weeks of life, could reduce perinatal HIV transmission by nearly 70\% [5]. A U. S. Public Health Service (USPHS) task force subsequently issued recommendations for the use of ZDV for reduction of perinatal HIV transmission [6] and universal prenatal HIV counseling and HIV testing with consent for all pregnant women in the United States [7]. Epidemiologic data have further confirmed the efficacy of ZDV for reduction of perinatal transmission, even among women with advanced disease, low $\mathrm{CD}^{+}{ }^{+} \mathrm{T}-$ lymphocyte counts, and prior ZDV therapy [8-13]. In addition, substantial advances have taken place in the treatment and monitoring of persons with HIV disease, which have resulted in changes in standard antiretroviral therapy for HIV infected adults. More aggressive combination drug regimens are now recommended to maximally suppress viral replication. For pregnant women, however, the benefits of antiretroviral therapy must be weighed against the risk of adverse events to the woman, fetus, and newborn; however pregnancy is generally not a reason to defer standard therapy. It should be offered with the addition of ZDV for prevention of perinatal HIV transmission to HIV infected pregnant women [4, 14].

For women with HIV infection, preconception care must focus on recommendations for safe sexual practices that prevent HIV transmission to sexual partners and protect women 
from acquiring potentially harmful sexually transmitted diseases or even additional strains of HIV that may be more virulent or resistant to therapy. Women also need education about perinatal transmission risks and prevention strategies, expectations for the child's future, and where desired, effective contraception until the optimal maternal health status for pregnancy is achieved, including appropriate therapy to maximally reduce viral load and optimize immune function. For HIV-infected women desiring pregnancy, specific counseling regarding available reproductive options that both prevent HIV exposure to uninfected partners, as well as preventing superinfection with resistant or more virulent virus should be discussed. The USPHS recommends that the following be included in preconception counseling for HIVinfected women: 1) selection of effective and appropriate contraceptive methods to reduce the likelihood of unintended pregnancy; 2) education and counseling about perinatal transmission risks, strategies to reduce those risks, and potential effects of HIV or treatment on pregnancy course and outcomes; 3) initiation or modification of antiretroviral therapy avoiding agents with potential toxicity for the developing fetus (e.g., efavirenz, hydroxyurea), choosing agents effective in reducing perinatal HIV transmission, attaining a stable, maximally suppressed maternal viral load, evaluating and controlling for therapy associated side effects which may adversely impact maternal-fetal health outcomes (e.g., hyperglycemia, anemia, hepatic toxicity); 4) evaluation and appropriate prophylaxis for opportunistic infections and administration of immunizations (e.g., influenza, pneumococcal, or hepatitis B vaccines) as indicated; 5) optimization of maternal nutritional status; 6 ) institution of the standard measures for preconception evaluation and management (e.g., assessment of reproductive and familial genetic history, screening for infectious diseases/sexually transmitted diseases, and initiation of folic acid supplementation); 7) screening for maternal psychological and substance abuse disorders, and 8) planning for perinatal consultation if desired or indicated [4]. The USPHS further notes that HIV infected women of childbearing potential receive primary healthcare services in various clinical settings, e.g., family planning, family medicine, internal medicine, obstetrics/gynecology, and stress that primary health-care providers should consider the fundamental principles of preconception counseling an integral component of comprehensive primary health care for improving maternal and child health outcomes [4].

The Infectious Disease Society of America (IDSA) recommends in-depth discussions about childbearing early in the course of HIV care for women who express a desire for a future pregnancy, who are not trying to conceive but are not using appropriate contraception consistently or who express uncertainty about reproductive plans. The goal of these discussions is to ensure informed decisions about contraception and to offer preconception counseling if pregnancy is desired.
IDSA recommends that providers regularly assess women's reproductive plans and their desire to have questions answered and, that providers question patients at each visit about interval menstrual history and sexual and contraceptive practices, offering pregnancy testing as indicated [15].

The American College of Obstetricians and Gynecologists (ACOG) and the American Academy of Pediatrics (AAP) recommend that screening for HIV infection be strongly recommended for women with unknown HIV status and who are considering pregnancy [16, 17]. The Centers for Disease Control and Prevention (CDC) is considering this recommendation for its revised recommendations for HIV testing in healthcare settings planned for 2006 [3]. In addition, these organizations as well as the Institute of Medicine and the U. S. Preventive Services Task Force recommend universal prenatal screening for HIV infection [3, 17-21], so that HIV-infected pregnant women can take full advantage of the available interventions to reduce perinatal transmission as well as to receive care for themselves. These organizations support an opt-out approach to HIV screening, (i.e., patients are notified that HIV testing will be done and consent is inferred unless testing is declined) [3] to normalize HIV testing in health care settings and to reduce the barriers clinicians experience with specific HIV test counseling and written informed consent.

Pre-test discussions regarding risk reduction for HIV infection are not a requirement for HIV testing and can be provided in the context of discussions of reproductive health and STD prevention. Routine HIV testing during preconception care can be integrated with other aspects of preconception care that require a blood sample and should not require substantial additional time commitments by providers. Improved implementation of preconception care will further extend the success in reducing perinatal HIV transmission in the U. S. as well as improve access to HIV testing and care to women. Because the issues accompanying HIV treatment during pregnancy and perinatal prophylaxis are rather complex, the case for preconception counseling and care for known HIV-infected women is clear.

\section{References}

1. Anderson RN, Smith BL. Deaths: leading causes for 2001. Nat Vital Stat Rep 2003;52(9):32-3, 53-4. Available at: http://www. cdc.gov/nchs/data/nvsr/nvsr52/nvsr52_09.pdf. Accessed Feb 3, 2006.

2. Anderson RN, Smith BL. Deaths: leading causes for 2002. Nat Vital Stat Rep 2005;53(17): 32-3, 53-4. Available at: http://www. cdc.gov/nchs/data/nvsr/nvsr53/nvsr53_17.pdf. Accessed Feb 3, 2006.

3. Mastro T. Normalizing HIV Testing in Health Care Settings. Oral presentation at the 13th Conference on Retroviruses and Opportunistic Infections; February, 2006; Denver, CO. Abstract 164.

4. Public Health Service Task Force recommendations for use of antiretroviral drugs in pregnant HIV-1-infected women for 
maternal health and interventions to reduce perinatal HIV-1 transmission in the United States. November 17, 2005. Available at: http://aidsinfo.nih.gov. Accessed February 3, 2006.

5. Connor EM, Sperling RS, Gelber R, et al. Reduction of maternalinfant transmission of human immunodeficiency virus type 1 with zidovudine treatment. N Engl J Med 1994;331:1173-80.

6. CDC. Recommendations of the Public Health Service Task Force on use of zidovudine to reduce perinatal transmission of human immunodeficiency virus. MMWR 1994;43(RR-11):1-20.

7. CDC. U. S. Public Health Service recommendations for human immunodeficiency virus counseling and voluntary testing for pregnant women. MMWR 1995;44 (RR-7):1-15.

8. Cooper ER, Nugent RP, Diaz C, et al. After AIDS Clinical Trial 076: the changing pattern of zidovudine use during pregnancy, and the subsequent reduction in vertical transmission of human immunodeficiency virus in a cohort of infected women and their infants. J Infect Dis 1996;174(6):1207-11.

9. Fiscus SA, Adimora AA, Schoenbach VJ, et al. Perinatal HIV infection and the effect of zidovudine therapy on transmission in rural and urban counties. JAMA 1996;275(19):1483-8.

10. Fiscus SA, Adimora AA, Funk ML, et al. Trends in interventions to reduce perinatal human immunodeficiency virus type 1 transmission in North Carolina. Pediatr Infect Dis J 2002;21(7):664-8.

11. Thomas P, Singh T, Bornschlegel K, et al. Use of ZDV to prevent perinatal HIV in New York City (NYC). 4th Conference on Retroviruses and Opportunistic Infections; January 22-261997; Washington, DC. Abstract 176.

12. Mayaux MJ, Teglas JP, Mandelbrot L, et al. Acceptability and impact of zidovudine for prevention of mother-to-child human immunodeficiency virus-1 transmission in France. J Pediatr 1997;131(6):857-62.
13. Harris NH, Thompson SJ, Ball R, et al. Zidovudine and perinatal human immunodeficiency virus type 1 transmission: a population-based approach. Pediatrics, 2002;109(4):e60. URL: http://www.pediatrics.org/cgi/content/full/109/4/e60.

14. Guidelines for Perinatal Care. 5th ed. Elk Grove Village, IL and Washington, DC: American Academy of Pediatrics and American College of Obstetricians and Gynecologists; 2002.

15. Aberg JA, Gallant JE, Anderson J, et al. Primary care guidelines for the management of persons infected with human immunodeficiency virus: recommendations of the HIV Medicine Association of the Infectious Diseases Society of America. Clin Infect Dis 2004;39:13.

16. American Academy of Pediatrics and American College of Obstetricians and Gynecologists. Joint statement of ACOG/AAP on Human immunodeficiency virus screening. 1999. Available at: http://www.acog.org/departments/dept_notice.cfm?recno = 18\&bulletin $=961$. Accessed Feb 3, 2006.

17. American College of Obstetricians and Gynecologists. Prenatal and perinatal human immunodeficiency virus testing: Expanded recommendations. Committee Opinion No. 304. Obstet Gynecol 2004;104:1119-24.

18. CDC. Revised recommendations for HIV screening of pregnant women. MMWR 2001;50(RR-19):63-85.

19. Institute of Medicine, National Research Council. Reducing the Odds: Preventing Perinatal Transmission of HIV in the United States. Washington, DC: National Academy Press; 1999.

20. U. S. Preventive Services Task Force. Screening for HIV: recommendation statement. Ann Intern Med 2005;143:32-7.

21. Chou R, Smits A, Hoyt Huffman L, et al. Prenatal screening for HIV: A review of the evidence for the U.S. Preventive Services Task Force. Ann Intern Med 2005;143:38-54. 